\title{
Using Mid-Sleep Time to Determine Chronotype in Young Adults with Insomnia-Related Symptoms
}

\author{
Sooyeon Suh, $\mathrm{PhD}^{1,2}$, Hyera Ryu, MA ${ }^{1}$, Soohyun Kim, BA ${ }^{1}$, Sujung Choi, $\mathrm{PhD}^{3,4}$, Eun Yeon Joo, MD, PhD ${ }^{4,5}$ \\ 'Department of Psychology, Sungshin Women's University, Seoul, Korea \\ 2Department of Psychiatry, Stanford University, Palo Alto, CA, USA \\ ${ }^{3}$ Department of Nursing, Samsung Medical Center, Department of Clinical Nursing Science, Graduate School of Clinical Nursing Science, \\ Sungkyunkwan University, Seoul, Korea \\ ${ }^{4}$ Department of Neurology, Neuroscience Center, Samsung Biomedical Research Institute, Samsung Medical Center, Sungkyunkwan University \\ School of Medicine, Seoul, Korea \\ ${ }^{5}$ Department of Health Sciences and Technology, SAIHST, Sungkyunkwan University, Seoul, Korea
}

Received: December 15, 2017

Revised: December 26, 2017

Accepted: December 27, 2017

Correspondence

Eun Yeon Joo, MD, $\mathrm{PhD}$

Department of Neurology,

Neuroscience Center,

Samsung Biomedical Research Institute,

Samsung Medical Center,

Sungkyunkwan University School of Medicine,

81 Irwon-ro, Gangnam-gu,

Seoul 06351, Korea

Tel +82-2-3410-3597

Fax +82-2-3410-005

E-mail ejoo@skku.edu

ORCID

Sooyeon Suh

https://orcid.org/0000-0003-0644-8634 Hyera Ryu

https://orcid.org/0000-0002-9962-0300

Soohyun Kim

https://orcid.org/0000-0002-7386-4753

Sujung Choi

https://orcid.org/0000-0003-2171-7441

Eun Yeon Joo

https://orcid.org/0000-0003-1233-959X
The Munich Chronotype Questionnaire (MCTQ) uses sleep behavior to assess chronotype, but the extent to which such sleep behavior based assessments might be useful in populations with sleep disorders, such as insomnia, is currently unclear. We thus systematically assessed sleep disorders, MCTQ and the Morningness-Eveningness Questionnaire (MEQ), another behavioral preferencebased assessment of chronotype, in 310 individuals. In a smaller substudy $(\mathrm{n}=121)$, we compared the MCTQ to sleep diaries in insomnia patients and good sleepers. Insomnia patients had overall lower consistency in chronotypes compared to good sleepers on the MCTQ compared to the MEQ, which was also evident when compared to sleep diaries. As insomnia disorder is characterized by time gaps spent awake during the night due to difficulty maintaining sleep, there may be limitations in using mid-sleep time as an accurate indicator of chronotype in insomnia patients. Our study suggests that the MCTQ should be used in conjunction with another existing questionnaire or assessment tool when assessing chronotype in insomnia patients.

Sleep Med Res 2017;8(2):107-111

Key Words Insomnia, Sleep disorder, Chronotype, Circadian rhythm.

\section{INTRODUCTION}

Insomnia is characterized by difficulty initiating and maintaining sleep that causes significant daytime distress and is prevalent in $13-33 \%$ of the general population. ${ }^{1}$ One characteristic that is closely associated with insomnia is chronotype. Morningness-Eveningness chronotype is defined as the preferred timing of the sleep-wake cycle. ${ }^{2}$ Morning types (M-types) prefer to go to sleep and wake up earlier and perform better in the mornings, whereas individuals who are evening types (E-types) prefer to go to sleep and get up later and performed better in the afternoon. ${ }^{3,4}$ Research indicates that approximately $25 \%$ of insomnia patients are later chronotypes. ${ }^{5}$ The close association between insomnia and chronotype makes it imperative to measure this behavioral dimension accurately.

One questionnaire that measures chronotype is the Munich ChronoType Questionnaire (MCTQ), developed by Roenneberg et al. ${ }^{6}$ which assesses actual sleep-wake timing and differentiates sleep-wake patterns between work days and free days and uses corrected mid-sleep time on free days after correcting for accumulated sleep debt over the week. The MCTQ provides information on sleep timing, including bedtime, sleep latency, sleep onset, offset and wake time considering work days and free days separately. Chronotype is assessed based on mid sleep time on free days. ${ }^{7}$ However, most of people tend to accumulate sleep debt on work days and compensate sleep on free days. ${ }^{6}$ Therefore, mid-sleep time on free days may overesti- 
mate chronotype. ${ }^{7}$ Thus, the MCTQ classifies chronotype based on mid-sleep on free days corrected for sleep debt on work days (MSFsc).

While the MCTQ has been widely used to determine chronotype in various populations, there have been relatively few studies investigating the accuracy of this measure in insomnia patients. Conceptually, using mid-sleep time as an indicator of chronotype may be less accurate in insomnia patients as insomnia is partly, by definition, characterized by difficulty maintaining sleep. Prolonged time spent in bed characterized by long periods of wakefulness after sleep onset would count towards mid-sleep time in insomnia patients, and thus may render using mid-sleep time as an indicator of chronotype inaccurate. Additionally, high day-to-day variability in sleep of insomnia patients may also be a factor that may contribute to the accuracy of using mid-sleep time to determine chronotype. ${ }^{8,9}$ In the past, most studies have used the Morningness-Eveningness Questionnaire (MEQ) to determine chronotype in insomnia patients, and while a few studies have used MCTQ with insomnia patients, ${ }^{10,11}$ they have not investigated the accuracy of chronotyping insomnia patients compared to good sleepers. Additionally, ascertaining chronotype may especially be difficult in insomnia patients with evening chronotype, which is especially true for many younger adults who have delayed preference for sleepwake timing.

Therefore, the current study was conducted to compare the MCTQ to the MEQ in insomnia patients and in good sleepers, and compare the MCTQ to sleep diaries in insomnia patients and good sleepers. We hypothesized that the MCTQ would be less accurate in reflecting chronotype for insomnia patients compared to good sleepers.

\section{METHODS}

All participants provided informed written consent prior to study participation. The study was approved by the local Institutional Review Board (SSWUIRB2015-057). Participants completed online questionnaires for this study.

\section{Participants}

Participants for this study were 499 individuals were recruited through brochures, class announcements, and online advertisements in the community. Among those recruited, 33 individuals were excluded due to missing MEQ data and 41 individuals were excluded because their responses did not reflect the intent of the questionnaires. For chronotype assessment, 106 participants who use an alarm clock on free days were excluded. Thus, 319 participants were used for final analysis in this study. Among these participants, $16.9 \%(\mathrm{n}=54)$ scored within the clinical insomnia range [scores higher than 15 on the Insomnia Severity Index (ISI)] and categorized as the "insomnia" group.
A small subset of participants $(n=121)$ that consisted of only females were selected to provide sleep diary information for 7 days for comparison with the MCTQ.

\section{Questionnaires}

\section{Munich Chronotype Questionnaire ${ }^{12}$}

The MCTQ was designed to measure actual sleep times separately for work and free days, and estimate chronotype based on the time-based variable (MSFsc) of the MCTQ. Mid-sleep time on free days corrected for sleep debt on work days (MSFsc) is a useful indicator to estimate chronotype. ${ }^{12}$ Before measuring chronotype, responses indicating use of an alarm clock on free days to wake up should be excluded from analyses. If sleep duration on work days were longer than or equal to sleep duration on free days, mid-sleep time on free days (MSF) was used as a marker for chronotype, and if sleep duration on work days were shorter than sleep duration on free days, we used MSFsc to measure chronotype (see equations below).

$\mathrm{MSF}=$ sleep onset + sleep duration $/ 2$

MSFsc $=$ MSF - (sleep duration on free days - average weekly sleep duration) $/ 2$

As suggested by Kühnle, ${ }^{13}$ MSFsc or MSF below 2.17 were classified as extreme M-types and MSFsc or MSF above 7.25 were classified as extreme E-types. These criteria were defined $2.5 \%$ at each end of the distribution as extreme chronotypes based on the distribution of MSFsc in their sample.

\section{Morningness-Eveningness Questionnaire ${ }^{14}$}

The MEQ is a 19-item self-report questionnaire designed to assess individual's chronotypes classified as M-type, intermediate-type (I-type) or E-type. It consists of mixed-format questions regarding the time in which individuals get up and go to bed, the preferred time to carry out physical and mental activity, and subjective alertness. The MEQ total score ranges from 16 to 86 , with scores above 58 classifying as M-types and scores below 41 as E-types. As suggested by Horne and Ostberg, ${ }^{14}$ scores above 71 were classified as extreme M-types and scores below 31 were classified as extreme E-types. Internal consistency (Cronbach's alpha) was 0.718 in the current sample.

\section{Insomnia Severity Index ${ }^{15}$}

The ISI is a 7-item self-report questionnaire that assesses the subjective symptoms and consequences of insomnia. ${ }^{15}$ Each item is scored on a $0-4$ likert scale, with total scores ranging from $0-28$. A higher score reflects more severe insomnia. Scores higher than 15 on the ISI indicate clinical insomnia. ${ }^{15}$ Internal consistency (Cronbach's alpha) was 0.769 in the current samples.

\section{7-day sleep diary}

A 7-day sleep diary was collected and the following variables were derived for analysis for both weekdays and weekends: la- 
tency to sleep onset (SOL), time awake after sleep onset (WASO), total sleep time (derived from bedtime, wake time, SOL, and WASO), and sleep efficiency (SE). Mid-sleep time was calculated by using the sleep onset and sleep offset time.

\section{Statistical Analysis}

Characteristics of MCTQ parameters were analyzed by descriptive statistics and independent sample t-test was used to compare MSFsc and MEQ scores in the insomnia group to the good sleepers. Chronotype classified by MEQ and MCTQ were presented using frequency analysis. Chronotype classification using MEQ and MCTQ scores were compared in insomnia patients and good sleepers based on various cut-offs for chronotype used in previous studies (using mid-sleep time $2.17 \%$ and $2.5 \%, 15 \%$ and $20 \%$ of extreme scores of the sample). ${ }^{13}$

Characteristics of sleep diary parameters were analyzed by descriptive statistics. Mann-Whitney U test was used to compare sleep diary parameters in insomnia patients and non-insomniacs. Correlation between MCTQ parameters [mid-sleep on work days (MSW), MSF, and MSFsc] and sleep diary parameters (MSW, MSF, and MSFsc using diary) was performed by Pearson correlation analysis. Additionally, correlation between mid-sleep time using MCTQ and sleep diary was compared in insomnia patients and good sleepers. SPSS software version 21.0 (SPSS Inc., IBM Corp., Armonk, NY, USA) was used for data analyses.

\section{RESULTS}

\section{Demographic Information}

A total of 319 participants participated in the study (mean age
$27.09 \pm 5.64 ; 78.1 \%$ female). Among these participants, $16.9 \%$ $(\mathrm{n}=54)$ scored within the clinical insomnia range (scores higher than 15 on the ISI) and categorized as the "insomnia" group. Demographic characteristics for good sleepers and the insomnia group are indicated in Table 1. The insomnia group had a significantly later mid-sleep time (indicated by MSFsc) compared to good sleepers $(5.77 \pm 1.66$ vs. $5.01 \pm 1.49$, p $<0.001)$. There was a statistical trend with the insomnia group showing slightly stronger eveningness tendencies with MEQ scores (44.15 \pm 8.52 vs. $46.48 \pm 8.35, \mathrm{p}=0.06$ ).

A subset of 121 participants selected from the original study completed 7 days of sleep diaries (mean age $20.93 \pm 1.73,100 \%$ females). Among these participants, $11.5 \%(\mathrm{n}=14)$ scored within the clinical insomnia range. Based on sleep diaries, the good sleeper group had significantly higher SE compared to the insomnia group $(90.39 \%$ vs. $83.54 \%, \mathrm{p}<0.05)$. There were no differences between other sleep parameters.

\section{MCTQ Parameters in Insomnia Patients Compared to Good Sleepers (Table 2)}

MEQ and MCTQ scores were compared in insomnia patients and good sleepers. The insomnia group consistently showed higher levels of inconsistency between the MEQ and MCTQ. When comparing various cut-off points, using 2.5\% of the MSFsc extreme scores from the current sample indicated highest rate of consistency compared to other criteria.

\section{Comparison of Mid-Sleep Time Using MCTQ vs. Sleep Diary in Insomnia Patients}

A comparison of sleep parameters based on sleep diaries revealed there were significant difference in WASO $(z=-2.87, \mathrm{p}<$ $0.01)$ and $\mathrm{SE}(\mathrm{z}=-2.86, \mathrm{p}<0.01)$ between insomnia patients

Table 1. Demographic characteristics per good sleepers and insomnia group

\begin{tabular}{|c|c|c|c|c|c|c|}
\hline \multirow{2}{*}{$\begin{array}{c}\text { Variable } \\
\text { names }\end{array}$} & \multicolumn{2}{|c|}{ Total } & \multicolumn{2}{|c|}{ Good sleepers } & \multicolumn{2}{|c|}{ Insomnia group } \\
\hline & n or mean & $\%$ or $\mathrm{SD}$ & n or mean & $\%$ or $\mathrm{SD}$ & n or mean & $\%$ or $\mathrm{SD}$ \\
\hline $\mathrm{n}$ & \multicolumn{2}{|c|}{319} & \multicolumn{2}{|c|}{263} & \multicolumn{2}{|c|}{54} \\
\hline Age & 27.09 & 5.64 & 27.41 & 5.66 & 25.38 & 5.32 \\
\hline \multicolumn{7}{|l|}{ Gender } \\
\hline Male & 70 & 21.9 & 63 & 24.0 & 7 & 13.0 \\
\hline Female & 249 & 78.1 & 200 & 76.0 & 47 & 87.0 \\
\hline
\end{tabular}

SD: standard deviation.

Table 2. Comparison of chronotype classification using MCTQ and MEQ in insomnia patients vs. good sleepers

\begin{tabular}{|c|c|c|c|c|c|c|c|c|c|c|c|c|}
\hline & \multicolumn{3}{|c|}{ MSFsc 2.17} & \multicolumn{3}{|c|}{ MSFsc $2.5 \%$} & \multicolumn{3}{|c|}{ MSFsc $15 \%$} & \multicolumn{3}{|c|}{ MSFsc $20 \%$} \\
\hline & No insomnia & Insomnia & $\mathrm{n}$ & No insomnia & Insomnia & $\mathrm{n}$ & No insomnia & Insomnia & $\mathrm{n}$ & No insomnia & Insomnia & $\mathrm{n}$ \\
\hline Inconsistency (\%) & 9.9 & 20.4 & 37 & 4.9 & 11.1 & 19 & 29.3 & 35.2 & 96 & 76.8 & 74.1 & 242 \\
\hline Consistency (\%) & 90.1 & 79.6 & 280 & 95.1 & 88.9 & 298 & 70.7 & 64.8 & 221 & 23.2 & 25.9 & 75 \\
\hline $\mathrm{n}$ & 263 & 54 & 317 & 263 & 54 & 317 & 263 & 54 & 317 & 263 & 54 & 317 \\
\hline
\end{tabular}

MCTQ: Munich Chronotype Questionnaire, MEQ: Morningness-Eveningness Questionnaire, MSFsc: mid-sleep on free days corrected for sleep debt on work days, MSFsc 2.17: MSFsc using mid-sleep time 2.17, MSFsc 2.5\%: MSFsc using 2.5\% of extreme scores of the sample, MSFsc 15\%: MSFsc using 15\% of extreme scores of the sample, MSFsc 20\%: MSFsc using 20\% of extreme scores of the sample. 
Table 3. Comparison of chronotype classification using MCTQ and MEQ in insomnia patients vs. good sleepers

\begin{tabular}{|c|c|c|c|c|c|c|c|c|c|c|c|c|}
\hline $\begin{array}{c}\text { Mid-sleep time } \\
\text { parameters }\end{array}$ & & MSW & & MSF & & MSFsc & & MSW_d & & MSF_d & & MSFsc_d \\
\hline \multicolumn{13}{|c|}{ Comparison of mid-sleep time using MCTQ vs. sleep diary in the total sample $(\mathrm{n}=121)$} \\
\hline MSW & & 1 & & & & & & & & & & \\
\hline MSF & & $0.461^{\dagger}$ & & 1 & & & & & & & & \\
\hline MSFsc & & $0.633^{\dagger}$ & & $0.891^{\dagger}$ & & 1 & & & & & & \\
\hline MSW_d & & $0.478^{\dagger}$ & & $0.504^{\dagger}$ & & $0.565^{\dagger}$ & & 1 & & & & \\
\hline MSF_d & & $0.339^{\dagger}$ & & $0.466^{\dagger}$ & & $0.466^{\dagger}$ & & $0.444^{\dagger}$ & & 1 & & \\
\hline MSFsc_d & & $0.354^{\dagger}$ & & $0.443^{\dagger}$ & & $0.477^{\dagger}$ & & $0.418^{\dagger}$ & & $0.941^{\dagger}$ & & 1 \\
\hline \multirow{2}{*}{$\begin{array}{c}\text { Mid-sleep time } \\
\text { parameters }\end{array}$} & \multicolumn{6}{|c|}{ No insomnia $(\mathrm{n}=107)$} & \multicolumn{6}{|c|}{ Insomnia $(\mathrm{n}=14)$} \\
\hline & MSW & MSF & MSFsc & MSW_d & MSF_d & MSFsc_d & MSW & MSF & MSFsc & MSW_d & MSF_d & MSFsc_d \\
\hline
\end{tabular}

Comparison of mid-sleep time using MCTQ vs. sleep diary in insomnia patients

\begin{tabular}{|c|c|c|c|c|c|c|c|c|c|c|c|c|}
\hline MSW & 1 & & & & & & 1 & & & & & \\
\hline MSF & $0.447^{\dagger}$ & 1 & & & & & 0.419 & 1 & & & & \\
\hline MSFsc & $0.663^{\dagger}$ & $0.861^{\dagger}$ & 1 & & & & $0.550^{*}$ & $0.966^{\dagger}$ & 1 & & & \\
\hline MSW_d & $0.514^{\dagger}$ & $0.433^{\dagger}$ & $0.533^{\dagger}$ & 1 & & & 0.367 & $0.622^{*}$ & $0.583^{*}$ & 1 & & \\
\hline MSF_d & $0.296^{\dagger}$ & $0.507^{\dagger}$ & $0.514^{\dagger}$ & $0.557^{\dagger}$ & 1 & & 0.493 & 0.228 & 0.246 & 0.159 & 1 & \\
\hline MSFsc_d & $0.331^{\dagger}$ & $0.470^{\dagger}$ & $0.516^{\dagger}$ & $0.529^{\dagger}$ & $0.946^{\dagger}$ & 1 & 0.508 & 0.322 & 0.348 & 0.160 & $0.918^{\dagger}$ & 1 \\
\hline
\end{tabular}

${ }^{*} \mathrm{p}<0.05,{ }^{\dagger} \mathrm{p}<0.01$

MSW: mid-sleep on work days, MSF: mid-sleep on free days, MSFsc: mid-sleep on free days corrected for sleep debt on work days, MSW_d: mid-sleep on work days using diary, MSF_d: mid-sleep on free days using diary, MSFsc_d: mid-sleep on free days corrected for sleep debt on work days using diary.

and good sleepers.

In the total sample, sleep diary parameters were used to calculate average 7-day mid-sleep time on work days and free days, and MSFsc, all of which were significantly correlated with MSW, MSF and MSFsc (all ps < 0.001). When comparing correlations between sleep diary and MCTQ parameters in insomnia patients with good sleepers, mid sleep time including MSW, MSF, and MSFsc using sleep diaries showed significant correlations with MSW, MSF, and MSFsc using MCTQ (all ps < 0.001) in the good sleeper group. However, in the insomnia group, MSW in the sleep diary was associated with MSF and MSFsc in the MCTQ ( $r=0.62, \mathrm{p}<0.05$, and $\mathrm{r}=0.58, \mathrm{p}<0.05$, respectively). All other correlations for mid-sleep time between the sleep diary and MCTQ parameters were not significant. Table 3 summarizes results.

\section{DISCUSSION}

The current study aimed to investigate how useful the MCTQ is to classify chronotype in insomnia patients compared to good sleepers. An important finding in our study was examining the accuracy of using the MCTQ in insomnia patients compared to good sleepers. Consistent with the overall sample, $2.5 \%$ yielded the best consistency with the MEQ (88.9\%) in insomnia patients compared to other cut-off points. However, when com- pared with good sleepers, insomnia patients had overall lower consistency in chronotypes compared to good sleepers, which was also evident when correlating with sleep diaries. While the good sleepers had significant correlations between sleep parameters on sleep diaries and the MCTQ, MSFsc was only significantly correlated with MSW on the sleep diary, and not with other parameters. Conceptually, considering that insomnia disorder is characterized by time gaps during the night due to difficulty maintaining sleep, there may be limitations when using mid-sleep time as an indicator of chronotype in insomnia patients. While the MCTQ does account for prolonged sleep onset latency, the questionnaire does not ask for wake after sleep onset, which is a common complaint among insomnia patients. While there have been a few studies investigating chronotype in insomnia patients, most of these studies have used the MEQ. ${ }^{16-18}$ While this is the first study investigating the utility of the MCTQ in insomnia patients, there have been increasingly more studies using the MCTQ to chronotype individuals due to the advantages of assessing work days and free days separately. Our study suggests that the MCTQ may have limitations when administered to insomnia patients, and should be used in conjunction with another existing questionnaire or assessment tool when categorizing chronotype in insomnia patients, especially when there is a high proportion of individuals with difficulty maintaining sleep. Future studies investigating this in a clinical population and other age groups are needed to further investigate 
this issue.

Despite our findings, this study had limitations. Considering the age range and higher proportion of females in our sample, it is difficult to generalize the results of this study as a general feature of insomnia. This study was conducted on a relatively narrow age range of participants, with young adults in their 20's and 30's. This resulted in a low frequency of M-types in our sample. Additionally, only a few number of individuals with insomnia completed the 7-day diary, which may have contributed to the low consistency of MCTQ. Finally, our study did not use biological or physiological markers of circadian rhythms, such as dim light melatonin onset, which may have been helpful in differentiating biological vs. preferential sleep-wake rhythm in both insomnia individuals and good sleepers.

In conclusion, the current study examined how accurate the MCTQ was in classifying chronotype in insomnia patients compared to good sleepers. When administering the MCTQ to insomnia patients compared to good sleepers, insomnia patients had overall lower consistency in chronotypes compared to good sleepers when compared to the MEQ and sleep diaries. As insomnia disorder is characterized by time gaps during the night due to difficulty maintaining sleep, there may be limitations in using mid-sleep time as an accurate indicator of chronotype in insomnia patients. Thus, the MCTQ may have limitations when administered to insomnia patients when categorizing chronotypes, and should be used in conjunction with another existing questionnaire or assessment tools.

\section{Acknowledgments}

We would like to thank Celine Vetter and Till Roenneberg for their help and consultation with this paper.

\section{Conflicts of Interest}

The authors have no financial conflicts of interest.

\section{Authors' Contribution}

Conceptualization: Eunyeon Joo, Sujung Choi, Sooyeon Suh. Data curation and data analysis: Hyera Ryu, Soohyun Kim. Methodology: Sooyeon Suh, Hyera Ryu, Soohyun Kim. Project administration: Sooyeon Suh, Eun Yeon Joo, Sujung Choi. Supervision: Eun Yeon Joo, Sooyeon Suh. Original draft: Sooyeon Suh, Hyera Ryu, Soohyun Kim. Review and editing: Sooyeon Suh, Hyera Ryu, Soohyun Kim, Sujung Choi, Eun Yeon Joo.

\section{REFERENCES}

1. Bartlett DJ, Marshall NS, Williams A, Grunstein RR. Sleep health New South Wales: chronic sleep restriction and daytime sleepiness. Intern
Med J 2008;38:24-31.

2. Duffy SJ, Keaney JF Jr, Holbrook M, Gokce N, Swerdloff PL, Frei B, et al. Short- and long-term black tea consumption reverses endothelial dysfunction in patients with coronary artery disease. Circulation 2001; 104:151-6.

3. Kerkhof GA. Inter-individual differences in the human circadian system: a review. Biol Psychol 1985;20:83-112.

4. Tankova I, Adan A, Buela-Casal G. Circadian typology and individual differences: a review. Pers Individ Dif 1994;16:671-84.

5. Ong JC, Huang JS, Kuo TF, Manber R. Characteristics of insomniacs with self-reported morning and evening chronotypes. J Clin Sleep Med 2007;3:289-94.

6. Roenneberg T, Kuehnle T, Juda M, Kantermann T, Allebrandt K, Gordijn M, et al. Epidemiology of the human circadian clock. Sleep Med Rev 2007;11:429-38.

7. Roenneberg T, Allebrandt KV, Merrow M, Vetter C. Social jetlag and obesity. Curr Biol 2012;22:939-43.

8. Suh S, Nowakowski S, Bernert RA, Ong JC, Siebern AT, Dowdle CL, et al. Clinical significance of night-to-night sleep variability in insomnia. Sleep Med 2012;13:469-75.

9. Bei B, Wiley JF, Trinder J, Manber R. Beyond the mean: a systematic review on the correlates of daily intraindividual variability of sleep/ wake patterns. Sleep Med Rev 2016;28:108-24.

10. Fossum IN, Nordnes LT, Storemark SS, Bjorvatn B, Pallesen S. The association between use of electronic media in bed before going to sleep and insomnia symptoms, daytime sleepiness, morningness, and chronotype. Behav Sleep Med 2014;12:343-57.

11. Sheaves B, Porcheret K, Tsanas A, Espie CA, Foster RG, Freeman D, et al. Insomnia, nightmares, and chronotype as markers of risk for severe mental illness: results from a student population. Sleep 2016;39:173-81.

12. Roenneberg T, Wirz-Justice A, Merrow M. Life between clocks: daily temporal patterns of human chronotypes. J Biol Rhythms 2003;18:8090.

13. Kühnle T. Quantitative analysis of human chronotypes [dissertation]. LMU München;2006. p.152.

14. Horne JA, Ostberg O. A self-assessment questionnaire to determine morningness-eveningness in human circadian rhythms. Int J Chronobiol 1976;4:97-110.

15. Bastien $\mathrm{CH}$, Vallières A, Morin CM. Validation of the Insomnia Severity Index as an outcome measure for insomnia research. Sleep Med 2001;2:297-307.

16. Saus E, Soria V, Escaramís G, Vivarelli F, Crespo JM, Kagerbauer B, et al. Genetic variants and abnormal processing of pre-miR-182, a circadian clock modulator, in major depression patients with late insomnia. Hum Mol Genet 2010;19:4017-25.

17. Drummond SP, Walker M, Almklov E, Campos M, Anderson DE, Straus LD. Neural correlates of working memory performance in primary insomnia. Sleep 2013;36:1307-16.

18. Steinan MK, Krane-Gartiser K, Langsrud K, Sand T, Kallestad H, Morken G. Cognitive behavioral therapy for insomnia in euthymic bipolar disorder: study protocol for a randomized controlled trial. Trials 2014;15:24. 